\title{
The Epistemic Value of the Living Fossils Concept
}

\author{
March 6, 2020
}

Word Count: 4,985

\begin{abstract}
Living fossils, taxa with similar members now and in the deep past, have recently come under scrutiny. Those who think the concept should be retained have argued for its epistemic and normative utility. This paper extends the epistemic utility of the living fossils concept to include ways in which a taxon's living fossil status can serve as evidence for other claims about that taxon. I will use some insights from developmental biology to refine these claims. Insofar as these considerations demonstrate the epistemic utility of the living fossils concept, they support retaining the concept and using it in biological research.
\end{abstract}

Living fossils are taxa in which extant organisms morphologically resemble fossilized organisms; paradigmatic examples include horseshoe crabs, coelacanths, and tuataras. Recently the living fossil concept has received considerable criticism, with even paradigmatic cases being contested. Some argue that the concept is not very useful for biologists, since these diverse cases are unlikely to be the product of unified phenomena, while others argue that the concept may be useful for certain epistemic and normative 
purposes. My aim in this paper is to address the epistemic value of living fossils. In particular, I will address the following question: Given that a taxon is a living fossil, what else do we know about it? Using considerations from developmental biology, I show that many common inferences from morphological similarity fail in the context of living

fossils. I will argue, however, that there are some inferences that are justified. I conclude that the living fossil concept has epistemic value, and hence should be retained.

After reviewing the recent literature (section 1), I will address three obvious conclusions that we might want to draw about living fossils (section 2): (1) non-morphological phenotypic similarity between the extant and past taxa, (2) the existence of a persistent lineage that includes these taxa, and (3) a slow rate of evolutionary change between these taxa. I will evaluate each of these inferences, especially using insights from developmental biology (section 3).

\section{Defining 'living fossil'}

Philosophers of biology have offered different characterizations of living fossils. Lidgard and Love (2018) argue for ways in which the concept is useful in setting research agendas, despite ambiguity in whether particular taxa should be classified as living fossils. Turner (2019) suggests an explicit definition of living fossil, one which he believes enables us to use living fossils to set conservation priorities. Specifically, Turner thinks that living fossils are taxa which have:

1. Prehistorically deep morphological stability,

2. Few extant species, and

3. High contribution to phylogenetic diversity. 
Werth and Shear (2014) give a similar characterization of living fossils, picking out "morphological conservatism" and "little taxonomic diversity" as relevant factors (434, 436).

Turner (2019) thinks there is epistemic value to the living fossil concept, including that "observations of [extant organisms in a living fossil taxon] can surely tell us something about the prehistoric ones" (11). The next two sections of this paper will specify exactly what we might be able to learn about these prehistoric taxa on the basis of their living fossil status. To sidestep debates about the specific definition of "living fossil," I will focus on the epistemic role of morphological similarity between past and extant taxa, a feature unanimously associated with living fossils.

Note that this paper is concerned with the possibility that the living fossil concept is epistemically valuable, although it may be valuable in other ways, including for normative purposes (as Turner 2019 argues). One way in which the living fossils concept might be epistemically valuable is that it helps us identify evolutionary episodes in need of explanation. Lidgard and Love (2018) think this is one purpose of the concept. In this case, a taxon's living fossil status, or at least the various features associated with that status, is the explanandum. However, in the remainder of this paper, I focus on another possible epistemic role for the living fossils concept to serve: a taxon's living fossil status can serve as evidence for other claims about the members of that taxon.

\section{Inferences from morphological similarity}

To reject the arguments of skeptics who think we should do away with the living fossil concept (e.g., Casane and Laurenti 2013, Mathers et al. 2013, Wagner et al. 2017), we 
should show what role the concept can play. Lidgard and Love and Turner recognize this, although they have different ideas of what this role is. However, the authors seem to be in agreement that part of what we want to be able to use the living fossil concept for is making inferences from the fact that past and extant taxa are morphologically similar to some other fact $F$ about these taxa. For short:

$$
\text { morphological similarity } \rightarrow F
$$

Both Lidgard and Love and Turner agree that we should be able to use the living fossil concept to make inferences of this form. Turner (2019) calls this the "epistemic value" of focusing on the morphological resemblance of past to extant taxa (11).

One possible fact $F$ that we may want to infer from morphological similarity between two taxa is that these taxa are phenotypically similar in ways above and beyond their morphological similarity. Take horseshoe crabs. Extant horseshoe crabs have hemocyanin in their blood (they use copper rather than iron to transport oxygen). Turner (2019) says, "the fossil record does not tell us that ancient horseshoe crabs had hemocyanin in their blood. But that seems like a fairly safe inference, given our background knowledge of phylogeny plus the observation that living ones do have hemocyanin in their blood" (11). The general type of inference that Turner is making is something like:

$$
\text { morphological similarity } \rightarrow \text { general phenotypic similarity }
$$

So far, I have been talking about morphological similarity, rather than morphological stability, the latter of which is used in Turner's definition. Turner (2019) says that showing morphological stability between past and extant taxa is equivalent to showing 
morphological similarity within a persistent lineage (3). If morphological similarity itself was evidence for persistence of a lineage, then morphological similarity would be evidence for morphological stability. In other words, the following inferences are equivalent:

$$
\text { morphologial similarity } \rightarrow \text { persistence of lineage }
$$

$$
\text { morphological similarity } \rightarrow \text { morphological stability }
$$

Finally, the living fossil concept may be useful for inferring rates of evolutionary change:

$$
\text { morphological similarity } \rightarrow \text { slow evolutionary rate }
$$

It only makes sense to talk about a rate of evolution within a given lineage, so inference 3 is necessary for inference 5 .

Inferences 2, 3, and 5 do not exhaust the possible inferences from morphological similarity to $F$ which we might make about living fossil taxa, but these examples show the possibility of making inferences about living fossil taxa based on what else we know about them. Thus these inferences provide good candidates if we want to demonstrate the epistemic utility of the living fossil concept.

The following section will use some insights from developmental biology to evaluate these inferences. 


\section{Developmental considerations}

Various concepts and theories in evolutionary biology have been revised in light of results in developmental biology. For example, developmental plasticity provides a possible explanation of speciation events, one compatible with the theory of punctuated equilibrium (West-Eberhard 2003). On the basis of such results, some have even suggested replacing the Modern Synthesis with the Extended Evolutionary Synthesis (e.g., Laland et al. 2015).

In this section, I will use some results in developmental biology to examine the arguments using morphological similarity from section 2 .

\subsection{Non-morphological phenotypic similarity}

Inference 2 says that we can infer from their morphological similarities that past and extant taxa have phenotypic similarities above and beyond these morphological similarities. For example, we would be able to infer the presence of hemocyanin in past horseshoe crabs on the basis that they are morphologically similar to extant horseshoe crabs.

Although I did not say this in section 2, one might have thought that the argument relating morphological similarity to general phenotypic similarity was implicitly assuming some relationship between morphological similarity and genetic similarity. If morphological similarity was good evidence for genetic similarity, and genetic similarity was good evidence for otherwise phenotypic similarity, then morphological similarity would be good evidence for phenotypic similarity. Including the implicit step, inference 2 would become: 
morphological similarity $\rightarrow$ genetic similarity $\rightarrow$ general phenotypic similarity

Even using a very rudimentary understanding of genetics, it is unlikely that inference 6 will work. The problem is that morphological similarity does not imply genetic similarity. There is not, in general, a one-to-one correspondence between genes and phenotypes, including morphology, so we can neither infer genetic information from phenotypic information nor vice versa. The same genes can result in different phenotypes, and the same phenotypes can be the result of different genes (e.g., Fusco and Minelli 2010). The former is the result of developmental plasticity, whereby a variety of environmental factors can affect phenotypic outcomes, for example by changing gene expression. The latter can be explained by the interchangeability of genes and environment in producing phenotypes,which West-Eberhard (2003) says "conflict[s] with the habit of supposing that the specificity of the [developmental] response comes entirely from the specificity of the gene" (117). If the argument from morphological similarity to general phenotypic similarity depends on an inference from morphological similarity to genetic similarity, the argument will fail, because the first half of inference 6 will turn out to be false. Additionally, and perhaps more intuitively, whatever genetic similarity might be implied by morphological similarity does not in itself imply the additional genetic similarity required to generate phenotypic similarity above and beyond morphology. In the case of the horseshoe crabs, different genes will be associated with morphology than with presence of hemocyanin.

However, morphological similarity may imply otherwise phenotypic similarity more 
directly, as indicated in the original inference 2, For instance, we might think that certain non-morphological phenotypes are strongly correlated with particular morphologies.

Whether this correlation is plausible is going to depend on the non-morphological phenotype. For instance, whether extant horseshoe crabs' blood is similar to past horseshoe crabs' will depend on whether features of blood are strongly correlated with morphology. If we had independent evidence that the contents of blood and an organism's morphology were strongly correlated, then the inference from the horseshoe crabs' morphology to their blood phenotype would be unproblematic. However, as Lidgard and Love (2018) say, "[r]etention of some phenotypic (traditionally morphological) characters does not adequately explain change or the lack thereof in other phenotypic characters" (766, emphasis in original). Fortey (2011) also thinks that there can be "no final proof one way or the other" about whether the past horseshoe crabs' blood contained hemocyanin (27).

In fact, developmental biologists have recently stressed the modularity of phenotypes. This refers to the separability of phenotypes, despite possible integration among them; developmental modules are semi-independent and dissociable, meaning that various traits can occur in different combinations in different organisms, with varying degrees of interdependence between different traits (West-Eberhard 2003, chpt. 4). These modules can then be selected for separately. For instance, terrestrial and arboreal salamanders have distinct foot morphology; the developmental pathways that lead to these differences are relatively independent from the salamanders' other traits, which go (more or less) unaffected (Gilbert 2000). This is made possible by the branching nature of development: cell differentiation occurs at branching decision points, which can be triggered by genetic or environmental switches. West-Eberhard (2019) says that 
modularity is a "universal property of organismic traits," because this branching process is ubiquitous (357). In the context of living fossils, and specifically inference 2 , modularity means that morphological similarity - which may be dissociable or independent from other phenotypes - does not provide adequate evidence for similarity of non-morphological traits.

Of course, some modules are more interdependent, and can be expected to co-occur. For example, morphology can constrain behavior such that particular behavioral traits are strongly correlated with particular morphological traits. Whether presence of one phenotype provides good evidence for presence of another phenotype depends on having independent evidence of the ways in which the different developmental modules may be interdependent.

Therefore, the wholesale inference from morphological similarity to phenotypic similarity above and beyond morphology is unlikely to be justified. This is not just a general skepticism about our ability to infer the presence of some traits from the observation of others; developmental modularity gives us good reason to believe that many traits are dissociable. More specific cases, where a correlation between morphology and other phenotypes is independently established, may allow for appropriate use of this inference in living fossil taxa. Indeed, Lidgard and Love (2018) suggest that one of the questions that research on living fossils might be able to answer has to do with the role of developmental modularity in patterns of evolutionary stasis (766). In other words, we may be able to come to a better understanding of the ways in which different traits are combined in developmental modules by studying stasis of these traits in living fossil taxa. 


\subsection{Persistence of lineage}

Inference 3 concludes on the basis of morphological similarity that the taxa are phylogenetically related such that they are both part of the same, persistent lineage, or, equivalently, that they are morphologically stable.

Note that neither Lidgard and Love nor Turner make the "lineage" relationship precise. Being part of the same lineage cannot require that the past fossil is an ancestor of the extant organisms, exactly, because we want to permit the past taxon and the extant one having an as-yet-unidentified common ancestor 11 Neither can the lineage relationship be as broad as a whole clade; it would become meaningless to differentiate living fossils from other cases of relatedness between past and extant taxa. Although it is beyond the scope of this paper to more precisely say what a lineage is, I take it that it is something between an ancestor-descendant relationship and a clade.

Setting this aside: does morphological similarity imply persistence of lineage or morphological stability?

As in the case of phenotypic similarity, perhaps there is an implicit assumption contained in inference 3 that involves a relationship between morphology and genetics. Inference 3 could be justified on the basis of this relationship: if morphological similarity implies genetic similarity, and genetic similarity implies the phylogenetic relationship that would hold within a persistent lineage, then morphological similarity would imply persistence of lineage. The resulting inference is:

\footnotetext{
${ }^{1}$ This is likely the case with horseshoe crabs - see Fortey $(2011)$.
} 
morphological similarity $\rightarrow$ genetic similiarity $\rightarrow$ persistence of lineage

I have already argued in section 3.1 that morphological similarity does not imply genetic similarity, so inference 7 will not work.

However, we should consider whether morphological similarity implies persistence of lineage without relying on a connection to genetic similarity. I will argue that there are several reasons to think that it does not; however, morphological similarity is often the best evidence we have of phylogenetic relationships.

First, morphological similarity and persistence of lineage do not exactly imply morphological stability, because there is the possibility that the morphological trait was lost and reemerged within the same lineage. Alternatively, if the past and extant taxa are in the same clade but do not have an ancestor-descendant relationship, then it may be possible that their common ancestor was not morphologically similar, in which case the morphology would have had to emerge separately on two different branches of the phylogenetic tree. This would be a case of convergent evolution, where the same traits evolve twice. These considerations when checking for morphological stability are the same as the well-known issues with testing for homology (similarity due to common ancestry) in general.

Developmental biologists point out that developmental pathways, even if not morphological traits, may be homologous (e.g., Nijhout 2019, 946). In these cases, which are called parallelism (rather than convergence), the trait may appear to evolve separately in two different branches, or may appear to be lost and reemerge, when in fact 
the mechanism by which the trait develops is actually homologous. It makes sense to broaden our concept of homology to include parallel evolution and recurrence of traits (West-Eberhard 2003, chpt. 25). I therefore concur with Turner (2019), although he does not explicitly use these developmental considerations to argue that morphological stability follows from morphological similarity.

Second, though, lack of morphological similarity may not be an indication of lack of morphological stability; polymorphism within a single species is relatively common. Two sample organisms from a species with morphologically distinct life stages may be mistaken as organisms belonging to different species if the organisms are observed in different of these life stages.$^{2}$ Extreme cases of sexual dimorphism are also liable to being mistaken for cases of multiple species. Note that both metamorphosis-induced life stages and sexual dimorphism may be the result of the developmental modularity discussed above (West-Eberhard 2003, 58, 75).

There is thus a risk of both false positives and false negatives in identifying persistence of lineage if we focus on morphological similarity. If there were a better indication of phylogenetic relationships than morphological similarity, we would use it instead.

These considerations notwithstanding, morphological similarity is often the best evidence we have for persistence of the same morphology over time, given that in the context of fossils we only have sporadic sample organisms and not any direct evidence of change over time $3^{3}$ This is part of the explanation for why the morphological species

${ }^{2}$ Turner (2016) acknowledges this point explicitly (64). See also Currie 2016.

${ }^{3}$ Note that our ability to acquire genetic information about fossil specimens may improve our epistemic position regarding phylogenetic relationships, if one thinks that the 
concept - rejected nearly unanimously as an adequate species concept for extant species - is still used by paleontologists (e.g., Turner 2011, 49-50 and Werth and Shear 2014, 442-43). Often the best evidence we have for phylogenetic relationships involving fossils is morphological similarities and differences, and persistence of lineage in the context of living fossils is no different.

\subsection{Evolutionary rates}

The third candidate inference we might want to make from morphological similarity within living fossil taxa is a slow rate of evolutionary change between the past and extant taxa. Recall that the argument for a slow rate of evolutionary change requires that we accept the inference to persistence of lineage. I have suggested that morphological similarity is often the best evidence we can hope to have for persistence of lineage. In this section I will assume that that inference is justified, and move on to examining inference 5, from morphological similarity to a slow rate of evolutionary change.

As in sections 3.1 and 3.2 , there is possibly an implicit assumption utilized here involving genetics. Let's ignore the possibility that the inference looks like this:

morphological similarity $\rightarrow$ genetic similarity $\rightarrow$ morphological stability $\rightarrow$ slow evolutionary rate

because we are assuming that morphological similarity is directly evidence for morphological stability (and I have already argued that morphological similarity does inference from genetic similarity to persistence of lineage is better than the inference from morphological similarity. See Jablonski and Shubin (2015). 
not imply genetic similarity). In this case, the implicit justification for 5 is instead that morphological stability implies genetic stability, which in turn implies a slow rate of evolutionary change:

morphological similarity $\rightarrow$ morphological stability $\rightarrow$ genetic stability $\rightarrow$ slow evolutionary rate

Many of the arguments I have given already that morphological similarity will not imply genetic similarity will be arguments against thinking that morphological stability implies genetic stability. I will not rehearse these arguments, because there is further reason to think that morphological stability does not imply genetic stability. Stabilizing selection acting on plastic traits can maintain the same phenotype over time, without necessarily having any effect whatsoever on rates of genetic change. For example, developmental plasticity is expected, especially in cases of extremely plastic traits like learning, to slow any directional increase or decrease in the propensity of a given phenotype in a population, because there is not ample opportunity for selection to act on any single phenotype (West-Eberhard 2003, 178). Furthermore, a process called "phenotypic accommodation" allows organisms to maintain functional phenotypic traits despite genetic mutation (West-Eberhard 2003, 51; see also West-Eberhard 2005).

The last step of inference 9- from genetic stability to slow rate of evolutionary change - is also problematic, although my critique here will be more controversial. An intuitive view is that a slow rate of evolutionary change in a lineage just is a slow rate of genetic change in that lineage, and that therefore the move from genetic stability to slow rate of evolution is unproblematic (e.g., Schopff 1984, Ho 2008). 
But is this really what we mean by slow rates of evolutionary change? Cases of stabilizing selection acting on phenotypes without causing a reduction in rates of genetic change show that it does not make sense to equate evolutionary change with genetic change. Traits on which stabilizing selection is acting should also be those traits which we say have a slow rate of evolutionary change: "the rate and degree of modification of a complex trait should be some positive function of its frequency of expression or use" (West-Eberhard 2003, 169). Traits with stability in a given lineage are exactly the traits with a slow rate of change. Therefore, there is no need to appeal to genetic stability to make the case for slow rates for evolutionary change - we can infer slow rates of evolutionary change directly from morphological stability.

Note that it is traits, and not lineages or taxa, to which we apply an evolutionary rate. Selection acts on phenotypes, not on organisms, species, or lineages. Lidgard and Love (2018) agree: "[c]haracters or character states are relatively more ancestral or derived, not whole organisms or lineages" (761, citing Omland, Cook, and Crisp 2008). Additionally, attribution of rates of change to traits rather than lineages is consistent with the idea of developmental modularity.

One of Turner's examples suggests that he thinks, in agreement with me, that morphological stability, the first feature in his definition of living fossils, is a better indication than molecular stability of slow rates of evolutionary change. Tuataras, a reptile from New Zealand, were thought to be living fossils on the basis of morphological stability, until researchers discovered that tuataras actually have a higher than average rate of molecular evolution (Hay et al. 2008). Some have used this result to criticize tuataras' status as a living fossil (e.g., Carnall 2016). Turner (2019)'s first criticism of this inference is that the Hay et al. (2008) study only uses mitochondrial DNA, which 
would not be expected to influence morphology (14). Turner's next point is more relevant for our purposes: he says that even if the study had used nuclear DNA, "developmental processes might insulate morphology from rapid molecular change" and that "[r]apid molecular change in the nuclear genome could also reflect selection pressures on aspects of the organism, like the immune system, that never show up in the fossil record" (14). These criticisms of the skeptics of tuataras' living fossil status line up nicely with my evaluation of the inference from morphological stability to genetic stability to a slow evolutionary rate. Turner concludes that "in spite of the high rate of molecular change, tuataras are a clear instance of a phylogenetic living fossil taxon" (15) $!^{4}$

However, Turner (2019) does not say that we can save the tuataras' living fossil status by appealing to a different idea of evolutionary rates. Indeed, his reconstruction of the argument against tuataras counting as living fossils is that "living fossils must have especially slow rates of evolutionary change, whereas the molecular evidence points toward especially rapid evolution in tuataras" (14). Turner's criticisms of this line of reasoning challenge the idea that a slow rate of evolution is a necessary feature of a living fossil taxa, rather than the idea that a slow rate of molecular change may not line up with a slow rate of (character) evolution at all. Later, in discussing coelacanths (another candidate for a living fossil taxon), Turner references "rates of morphological change," but does not equate these rates with rates of evolutionary change (16). Indeed, Turner says that "morphological stability in certain characters is entirely compatible with evolutionary change happening under the geological radar" (18). However, as I have

\footnotetext{
${ }^{4}$ Interestingly, Hay et al. (2008) also interpret their results about the faster-thanexpected rate of molecular evolution in tuataras as being evidence that "rates of neutral molecular and phenotypic evolution are decoupled" (106).
} 
argued, morphological stability in certain characters is exactly not compatible with evolutionary change happening on those characters. My suspicion is that Turner is confusing rates of evolutionary change with rates of molecular change here.

Werth and Shear (2014) have a similar take on the case of tuataras. While Werth and Shear do not think that evidence of a higher rate of molecular evolution in this lineage disqualifies it as a living fossil taxon, they say that the high molecular rates "provide strong evidence countering the misconception that living fossils have stopped evolving" (438). In other words, Werth and Shear - like Turner - apparently want to maintain the tuataras' status as a living fossil by arguing that living fossils need not have a slow rate of evolutionary change, rather than by claiming that rates of evolutionary change are best measured at the level of traits and not genes, necessarily (although insofar as genes are themselves traits, a rate of evolution could apply to them as well).$^{5}$

One implication of focusing on the inference from morphological stability to slow rates of evolutionary change is that it is not clear what the epistemic role evidence of molecular stability in a lineage could have. Lidgard and Love (2018) say, "the primary role of the living fossil concept is to mark out more precisely what requires explanation in a given instance for a particular entity in order to account for morphological and molecular stability or persistence over long periods of evolutionary time" (763, emphasis added). If molecular stability does not let us infer an evolutionary rate (other than an evolutionary rate at the molecular level itself), then why might we want to know about

\footnotetext{
${ }^{5}$ Note that Werth and Shear do acknowledge that "Some biologists speculate that mere genetic change does not translate to evolutionary change" and that there is "independence between molecular and morphological evolution," although they do not endorse this position (439).
} 
molecular stability at all? Lidgard and Love have (at least) one interesting response: we might want to know how molecular and morphological rates of change are related or decoupled (766).

It is especially interesting that the inference to slow evolutionary rates from morphological similarity is the most secure of those I have considered in this paper, because Darwin (1859/1964)'s use of the term "living fossils" was in the context of explaining why some lineages display slower rates of evolutionary change than others. While Darwin's explanation was that these lineages had been "exposed to less severe competition" (107), and now we know that the reasons for stabilizing selection are more complicated, he still made, by my account, the most reasonable inference from the morphological similarity of extant and past taxa.

The various attempted inferences and critiques of these inferences examined in this section are summarized in table 1 .

\section{Conclusion}

This paper's primary contribution has been to disambiguate the inferences that we can justifiably make on the basis of classifying a taxon as a living fossil. In doing so, I have specified some of the ways in which the living fossil concept may be epistemically useful. This adds to claims that the living fossil concept is epistemically useful in other ways, such as by identifying phenomena in need of explanation. I also intend to complement, not supplant, accounts in which the living fossil concept is useful for non-epistemic 


\begin{tabular}{|l|l|l|}
\hline$F$ & Inference & Evaluation \\
\hline \hline $\begin{array}{l}\text { General pheno- } \\
\text { typic similarity }\end{array}$ & $\begin{array}{l}\text { morphological similarity } \rightarrow \\
\text { genetic similarity } \rightarrow \text { general } \\
\text { phenotypic similarity }\end{array}$ & $\begin{array}{l}\text { Morphological similarity does not } \\
\text { imply genetic similarity }\end{array}$ \\
\hline $\begin{array}{l}\text { General pheno- } \\
\text { typic similarity }\end{array}$ & $\begin{array}{l}\text { morphological similarity } \rightarrow \\
\text { general phenotypic similar- } \\
\text { ity }\end{array}$ & $\begin{array}{l}\text { Morphological similarity only im- } \\
\text { plies phenotypic similarity for some } \\
\text { phenotypes (developmental modu- } \\
\text { larity) }\end{array}$ \\
\hline $\begin{array}{l}\text { Persistence of lin- } \\
\text { eage }\end{array}$ & $\begin{array}{l}\text { morphological similarity } \rightarrow \\
\text { genetic similarity } \rightarrow \text { persis- } \\
\text { tence of lineage }\end{array}$ & $\begin{array}{l}\text { Morphological similarity does not } \\
\text { imply genetic similarity }\end{array}$ \\
\hline $\begin{array}{l}\text { Persistence of lin- } \\
\text { eage }\end{array}$ & $\begin{array}{l}\text { morphological similarity } \rightarrow \\
\text { persistence of lineage }\end{array}$ & $\begin{array}{l}\text { Morphological similarity does not } \\
\text { imply persistence of lineage, but it } \\
\text { might be the best evidence we have }\end{array}$ \\
\hline $\begin{array}{l}\text { Slow evolutionary } \\
\text { rate }\end{array}$ & $\begin{array}{l}\text { morphological similarity } \rightarrow \\
\text { morphological stability } \rightarrow \\
\text { genetic stability } \rightarrow \text { slow evo- } \\
\text { lutionary rate }\end{array}$ & $\begin{array}{l}\text { Morphological stability does not } \\
\text { imply genetic stability, and genetic } \\
\text { stability does not imply a slow evo- } \\
\text { lutionary rate }\end{array}$ \\
\hline $\begin{array}{l}\text { Slow evolutionary } \\
\text { mote }\end{array}$ & $\begin{array}{l}\text { Morphological similarity } \rightarrow \\
\text { slow evolutionary rate }\end{array}$ & $\begin{array}{l}\text { a slow evolutionary rate, relative to } \\
\text { that morphology }\end{array}$ \\
\hline
\end{tabular}

Table 1: Summary.

reasons. One possible area for future research is identifying the ways in which the epistemic and non-epistemic uses of the concept may interact. For instance, Turner thinks that a living fossil taxon's high contribution to phylogenetic diversity has implications for conservation efforts. But we may need to address epistemic issues before we are able to draw appropriate normative conclusions.

This paper has also served as an example of how developmental biology can be useful for paleontologists. Historically, development hasn't been given much consideration in making claims about fossils, largely because fossil evidence does not include information about developmental processes. Discussions of homology in general, which are relevant 
to persistence of lineage, involve the contributions of both paleontologists and developmental biologists. Living fossils serve as another good example for how considerations from developmental biology and paleontology could be productively combined, because we have evidence about fossilized as well as living taxa. The arguments I have made in this paper, such as those regarding developmental modularity, may have other implications for paleontology outside of the context of living fossils, and more generally point to the fertility of of exploring the intersection between developmental biology and paleontology. 


\section{References}

Carnall, M. 2016. "Let's make living fossils extinct". The Guardian. https://www.theguardian.com/science/2016/jul/06/why-its-time-to-makeliving-fossils-extinct.

Casane, D., and P. Laurenti. 2013. "Why coelacanths are not "living fossils"”. Bioessays $35: 332-338$.

Currie, A. M. 2016. "The mystery of the triceratops mother: How to be a realist about the species category". Erkenn 81:785-816.

Darwin, C. 1859/1964. On the origin of species. Cambridge, MA: Harvard University Press.

Fortey, R. 2011. Horseshoe crabs and velvet worms: The story of the animals and plants that time has left behind. New York, NY: Vintage Books.

Fusco, G., and A. Minelli. 2010. "Phenotypic plasticity in development and evolution: facts and concepts". Philosophical Transactions of the Royal Society B 365:547-556.

Gilbert, S. F. 2000. Developmental Biology. Sunderland, MA: Sinauer Associates.

Hay, J. M., et al. 2008. "Rapid molecular evolution in a living fossil". Trends Genet 24 (3): 106-109.

Ho, S. 2008. "The molecular clock and estimating species divergence". Nature education $1(1): 168$.

Jablonski, D., and N. H. Shubin. 2015. "The future of the fossil record: Paleontology in the 21st century". PNAS 112 (16): 4852-4858. 
Laland, K. N., et al. 2015. "The extended evolutionary synthesis: its structure, assumptions and predictions". Proc. R. Soc. B. 282:20151019.

Lidgard, S., and A. C. Love. 2018. "Rethinking living fossils". Bioscience 68 (10): $760-770$.

Mathers, T.C., et al. 2013. "Multiple global radiations in tadpole shrimps challenge the concept of "living fossils"'. Peer $J$ 1:e62.

Nijhout, H. F. 2019. "The multistep morphing of beetle horns: Genes that specify insect wings initiate horn development in dung beetles". Science 366 (6468): 946-947.

Omland, K. E., L. G. Cook, and M. D. Crisp. 2008. "Tree thinking for all biology: The problem with reading phylogenies as ladders of progress". BioEssays 30:854-867.

Schopff, T. J. M. 1984. "Rates of evolution and the notion of "living fossils"". Ann. Rev. Earth Planet. Sci. 12:245-292.

Turner, D. D. 2016. "A second look at the color of dinosaurs". Studies in History and Philosophy of Science 55:60-68.

— . 2019. "In Defense of Living Fossils". Biology 8 Philosophy 34 (23).

— . 2011. Paleontology: a philosophical introduction. New York, NY: Cambridge University Press.

Wagner, P., et al. 2017. "Ontogenetic sequence comparison of extant and fossil tadpole shrimps: no support for the "living fossil" concept". PalZ 91:463-472.

Werth, A. J., and W. A. Shear. 2014. "The evolutionary truth about living fossils". American Scientist 102:434-443. 
West-Eberhard, M. J. 2003. Developmental Plasticity and Evolution. New York, NY: Oxford University Press.

— . 2019. "Modularity as a universal emergent property of biological traits". Journal of Experimental Zoolology 332:356-364.

— . 2005. "Phenotypic Accommodation: Adaptive Innovation Due to Developmental Plasticity". Journal of experimental zoology 304B:610-618. 